All authors: Memorial Sloan Kettering Cancer Center and Weill Cornell Medical College, New York, NY.

Published online ahead of print at www.jco.org on January 20, 2015.

Authors' disclosures of potential conflicts of interest are found in the article online at www.jco.org. Author contributions are found at the end of this article.

Corresponding author: Jedd D. Wolchok, MD, PhD, Memorial Sloan Kettering, Cancer Center, 1275 York Ave, New York, NY 10065; e-mail: wolchokj@mskcc.org.

(c) 2015 by American Society of Clinica Oncology

0732-183X/15/3317w-1974w/\$20.00

DOI: $10.1200 / J C O .2014 .59 .4358$

\title{
Immune Checkpoint Blockade in Cancer Therapy
}

Michael A. Postow, Margaret K. Callahan, and Jedd D. Wolchok

$$
\begin{array}{llllllll}
\text { A } & \text { B } & \text { S } & \text { T } & \text { R } & \text { A } & \text { C } & \text { T }
\end{array}
$$

Immunologic checkpoint blockade with antibodies that target cytotoxic T lymphocyte-associated antigen 4 (CTLA-4) and the programmed cell death protein 1 pathway (PD-1/PD-L1) have demonstrated promise in a variety of malignancies. Ipilimumab (CTLA-4) and pembrolizumab (PD-1) are approved by the US Food and Drug Administration for the treatment of advanced melanoma, and additional regulatory approvals are expected across the oncologic spectrum for a variety of other agents that target these pathways. Treatment with both CTLA-4 and PD-1/PD-L1 blockade is associated with a unique pattern of adverse events called immune-related adverse events, and occasionally, unusual kinetics of tumor response are seen. Combination approaches involving CTLA-4 and PD-1/PD-L1 blockade are being investigated to determine whether they enhance the efficacy of either approach alone. Principles learned during the development of CTLA-4 and PD-1/PD-L1 approaches will likely be used as new immunologic checkpoint blocking antibodies begin clinical investigation.

\section{J Clin Oncol 33:1974-1982. (C) 2015 by American Society of Clinical Oncology}

\section{INTRODUCTION}

The immune system plays an important role in controlling and eradicating cancer. Nevertheless, in the setting of malignancy, multiple mechanisms of immune suppression may exist that prevent effective antitumor immunity. Antibody therapy directed against several negative immunologic regulators (checkpoints) is demonstrating significant success and is likely to be a major component of treatment for patients with a variety of malignancies.

This review is focused on antibodies that block cytotoxic T lymphocyte-associated antigen 4 (CTLA-4) and the programmed cell death protein 1 pathway (PD-1/PD-L1). We discuss the preclinical rationale and clinical experience with these antibodies and then review the unique considerations relevant for treating patients with these agents.

\section{CTLA-4 PRECLINICAL RATIONALE AND} CLINICAL EFFICACY

CTLA-4 was the first immune checkpoint receptor to be clinically targeted (Fig 1) Normally, after T-cell activation, CTLA-4 is upregulated on the plasma membrane where it functions to downregulate T-cell function through a variety of mechanisms, including preventing costimulation by outcompeting CD28 for its ligand, B7, and also by inducing T-cell cycle arrest. ${ }^{1-5}$ Through these mechanisms and others, CTLA-4 has an essential role in main- taining normal immunologic homeostasis, as evidenced by the fact that mice deficient in CTLA- 4 die from fatal lymphoproliferation. ${ }^{6,7}$ Recognizing the role of CTLA- 4 as a negative regulator of immunity, investigators led studies demonstrating that antibody blockade of CTLA-4 could result in antitumor immunity in preclinical models. ${ }^{8,9}$

On the basis of this preclinical rationale, two antibodies targeting CTLA-4, ipilimumab (BristolMyers Squibb, Princeton, NJ) and tremelimumab (formerly Pfizer, currently MedImmune/AstraZeneca, Wilmington, DE), entered clinical development. Early reports of both agents showed durable clinical responses in some patients. ${ }^{10-12}$ Unfortunately, despite a proportion of patients experiencing a durable response, tremelimumab did not statistically significantly improve overall survival, which led to a negative phase III study comparing tremelimumab to dacarbazine/ temozolomide in patients with advanced melanoma. ${ }^{13}$ It is possible that the lack of an overall survival benefit was a result of the crossover of patients treated with chemotherapy to an expanded access ipilimumab program or a result of the dosing or scheduling considerations of tremelimumab.

Ipilimumab, however, was successful in improving overall survival in two phase III studies involving patients with advanced melanoma. ${ }^{14,15}$ Although the median overall survival was only improved by several months in each of these studies, landmark survival after treatment initiation favored ipilimumab; in the first phase III study, $18 \%$ of patients were alive after 2 years compared with $5 \%$ of 


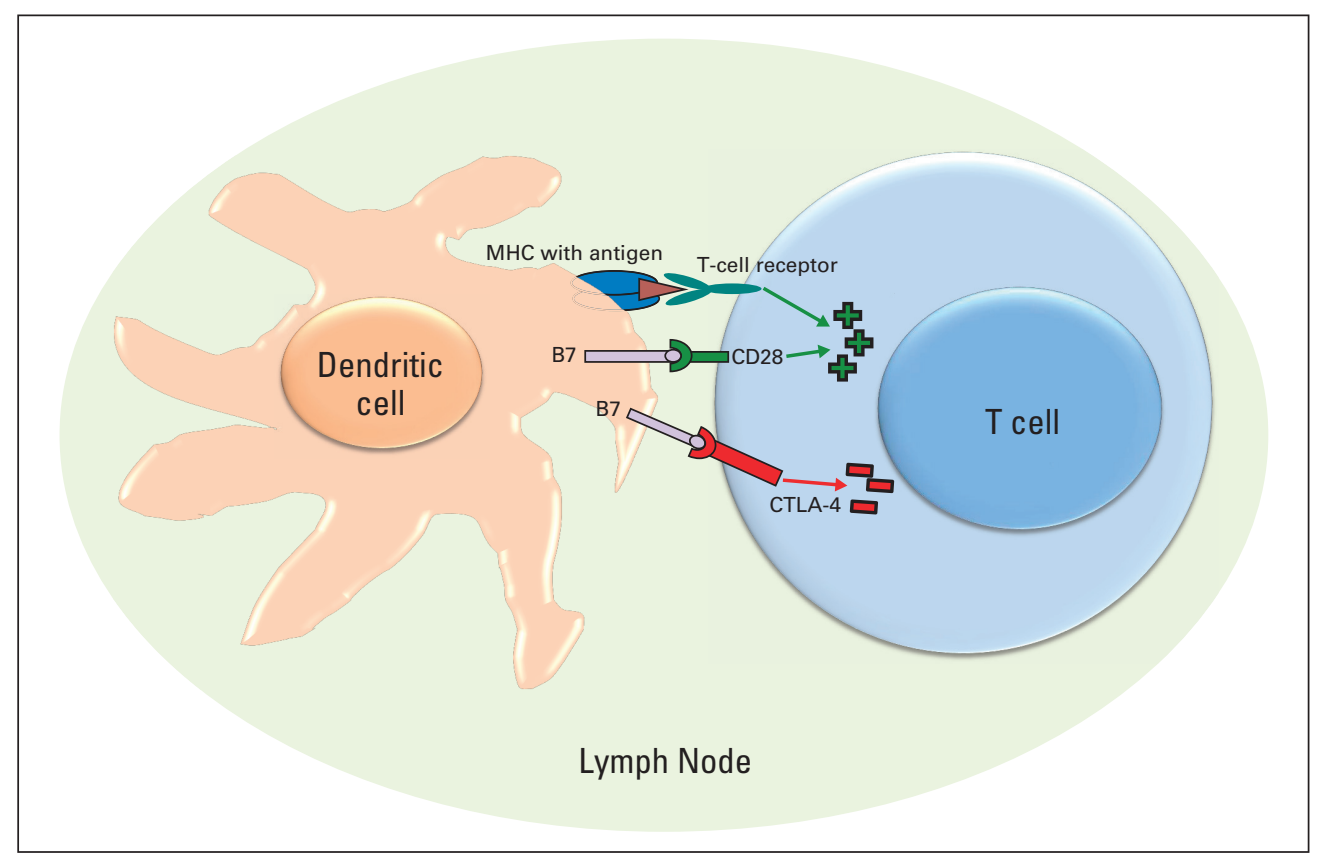

Fig 1. The cytotoxic T lymphocyte-associated antigen 4 (CTLA-4) immunologic checkpoint. T-cell activation requires antigen presentation in the context of a major histocompatibility complex (MHC) molecule in addition to the costimulatory signal achieved when B7 on an antigen-presenting cell (dendritic cell shown) interacts with CD28 on a T cell. Early after activation, to maintain immunologic homeostasis, CTLA-4 is translocated to the plasma membrane where it downregulates the function of $\mathrm{T}$ cells.

patients who received the control treatment of gp100 vaccination. ${ }^{14}$ More recently reported pooled data from clinical trials of ipilimumab confirm that approximately $20 \%$ of patients will have long-term survival of at least 3 years after ipilimumab therapy, with the longest reported survival reaching 10 years. ${ }^{16-18}$

For patients with other malignancies, CTLA-4 antibody therapy has also shown some benefits. Ipilimumab, in combination with carboplatin and paclitaxel in a phased treatment schedule, showed improved progression-free survival compared with carboplatin and paclitaxel alone for patients with non-small-cell lung cancer. ${ }^{19}$ Several patients with pancreatic cancer had declines in CA 19-9 when ipilimumab was given with GVAX (Aduro, Berkeley, CA), ${ }^{20}$ and ipilimumab has also resulted in responses in patients with prostate cancer. ${ }^{21}$ Unfortunately, a phase III study in patients with castrateresistant prostate cancer who experienced progression on docetaxel chemotherapy demonstrated that after radiotherapy, ipilimumab did not improve overall survival compared with placebo. $^{22}$ Although this study is felt to have been a negative study, ipilimumab may have conferred a benefit to patients with favorable prognostic features, such as the absence of visceral metastases, but this requires further study. Another CTLA-4-blocking antibody, tremelimumab, has shown responses in patients with mesothelioma, and ongoing trials are under way. ${ }^{23}$

\section{PD-1 PRECLINICAL RATIONALE AND CLINICAL EFFICACY}

Success targeting CTLA-4 has created enthusiasm for clinical approaches targeting other immunologic checkpoints, namely PD-1/ PD-L1 (Fig 2). PD-1 is a negative regulator of T-cell activity that limits the activity of $\mathrm{T}$ cells at a variety of stages of the immune response when it interacts with its two ligands PD-L1 and PD-L2. ${ }^{24-26}$ When engaged by ligand, through phosphatase activity, PD-1 inhibits kinase signaling pathways that normally lead to T-cell activation. ${ }^{25}$ Mice deficient in PD-1 have a distinct autoimmune phenotype from mice deficient in CTLA-4. ${ }^{27,28}$ Perhaps this finding is unsurprising because, unlike CTLA-4, which is primarily believed to regulate immune responses early in T-cell activation, PD-1 is primarily believed to inhibit effector T-cell activity in the effector phase within tissue and tumors. ${ }^{29} \mathrm{PD}-1$ is expressed on many immunologic cells, including B cells and natural killer cells, and therapeutic blockade of the PD-1 pathway may influence the function of these cells as well. ${ }^{29,30}$

A number of antibodies that disrupt the PD-1 axis have entered clinical development. Although the various antibodies differ in structure (Table 1), they can largely be broken down into the following two main categories: those that target PD-1 (nivolumab, Bristol-Myers Squibb; pembrolizumab, Merck, Whitehouse Station, NJ; pidilizumab, CureTech, Yavne, Israel) and those that target PD-L1 (MPDL3280A, Genentech, South San Francisco, CA; MEDI4736, MedImmune/AstraZeneca; BMS-936559, Bristol-Myers Squibb; MSB0010718C, EMD Serono, Rockland, MA). AMP-224 (Amplimmune, Gaithersburg, MD/GlaxoSmithKline, Philadelphia, PA) is a PD-L2 fusion protein that does not directly target PD-1 or PD-L1, but instead is believed to deplete PD-1-positive T cells. ${ }^{31}$

Both nivolumab and pembrolizumab have demonstrated highly durable response rates with minimal toxicity in large phase I studies involving patients with advanced melanoma, non-small-cell lung cancer, renal cell carcinoma, and other solid tumors. ${ }^{32-34}$ Although initial enthusiasm for PD-1 blockade was generated from large phase I studies, randomized phase III data in patients with melanoma are now emerging. In one study of patients with melanoma who had experienced progression on ipilimumab, nivolumab resulted in a $32 \%$ overall response rate compared with 11\% for chemotherapy (dacarbazine or carboplatin/paclitaxel) with less frequent high-grade treatmentrelated adverse events. ${ }^{35}$ A separate phase III study testing nivolumab against dacarbazine chemotherapy in patients with advanced melanoma was stopped early by an independent data monitoring committee because patients treated with nivolumab had improved overall survival compared with patients treated with dacarbazine. 


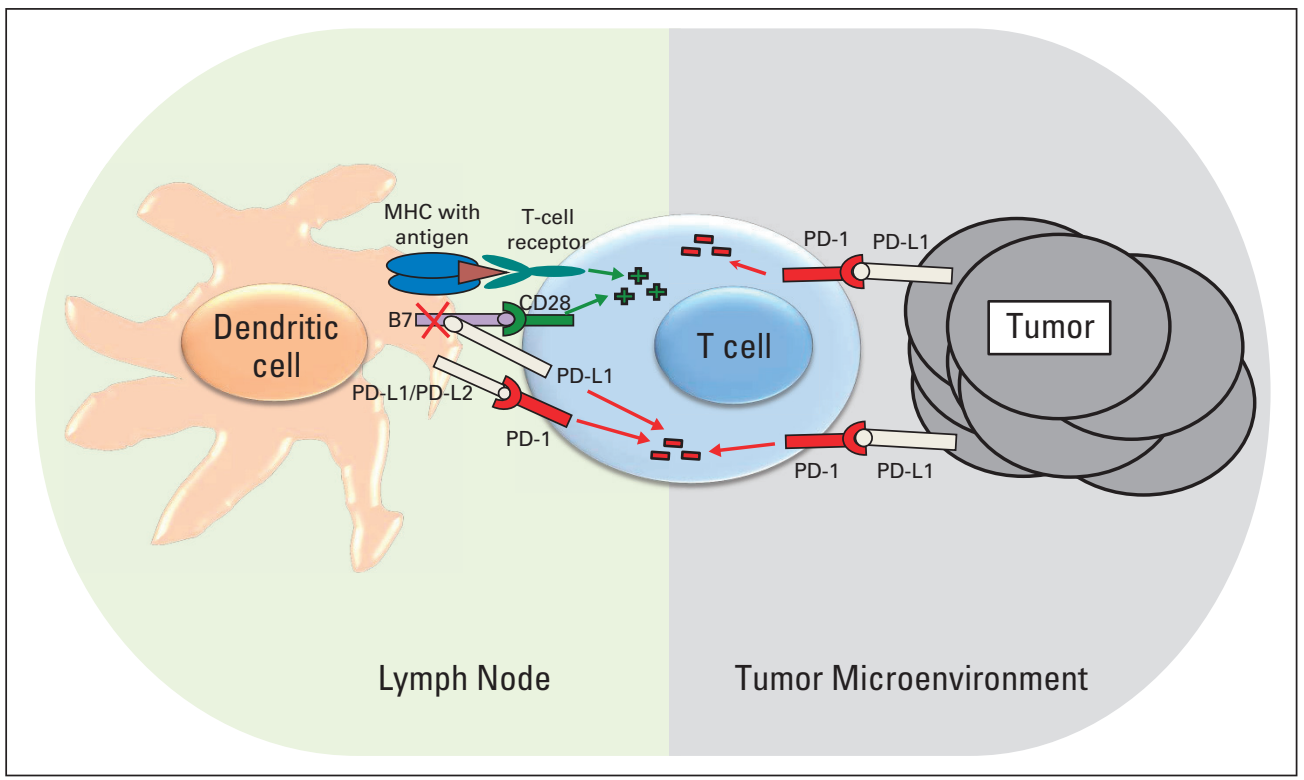

Fig 2. The programmed cell death protein 1 (PD-1) immunologic checkpoint. PD-1 is expressed on activated T cells. Interactions between PD-1 and its ligands, PD-L1 and PD-L2, are complex and occur at multiple steps of an immune response. For illustration, we have shown an interaction early after activation in the lymph node where PD-L1/PD-L2 on an antigenpresenting cell (dendritic cell shown) negatively regulates $\mathrm{T}$-cell activity through PD-1 and through an interaction between B7 and PD-L1. The PD-1 pathway is also likely important in the tumor microenvironment where PD-L1 expressed by tumors interacts with PD-1 on T cells to suppress T-cell effector function. $\mathrm{MHC}$, major histocompatibility complex.

Pembrolizumab has similarly been shown to result in impressive tumor responses and was recently approved by the US Food and Drug Administration for patients with melanoma previously treated with ipilimumab and, if relevant, a BRAF inhibitor. ${ }^{36} \mathrm{Al}-$ though nivolumab and pembrolizumab have predominantly demonstrated activity in solid tumors, pidilizumab has mostly been clinically evaluated in hematologic malignancies, with responses seen as monotherapy and in combination regimens with rituximab. ${ }^{37-39}$

Targeting PD-L1 is a similarly promising approach to targeting PD-1. Targeting PD-L1, however, may result in different biologic effects than targeting PD-1. In addition to binding PD-1, PD-L1 also is believed to exert negative signals on T cells by interacting with $\mathrm{B} 7 .^{40}$ PD-L1-blocking antibodies prevent this interaction, but PD-1blocking antibodies do not. Another slight difference is that PD-L1 antibodies do not prevent PD-1 from interacting with PD-L2, although the effect of this interaction remains unknown.

BMS-956559 was the first PD-L1 antibody to show objective tumor responses in patients with a variety of solid tumors. ${ }^{41}$

\begin{tabular}{|c|c|}
\hline Target and Agent & Class \\
\hline \multicolumn{2}{|l|}{$\overline{\mathrm{PD}-1}$} \\
\hline Nivolumab (MDX1106, BMS-936558) & IgG4 fully human Ab \\
\hline Pembrolizumab (MK-3475) & IgG4 engineered humanized $A b$ \\
\hline Pidilizumab (CT-011) & $\lg \mathrm{G} 1$ humanized $\mathrm{Ab}$ \\
\hline \multicolumn{2}{|l|}{ PD-L1 } \\
\hline BMS935559 (MDX-1105) & IgG4 fully human $\mathrm{Ab}$ \\
\hline MPDL3280A & IgG1 engineered fully human $A b$ \\
\hline MEDI4736 & $\lg \mathrm{G} 1$ engineered fully human $\mathrm{Ab}$ \\
\hline MSB0010718C & IgG1 fully human Ab \\
\hline \multicolumn{2}{|l|}{ PD-1-positive T cells } \\
\hline AMP-224 & Fc of human IgG-PD-L2 fusion \\
\hline
\end{tabular}

MPDL3280A, MEDI4736, and MSB0010718C have also been shown to result in disease responses in early-phase clinical trials in a number of malignancies including tumor types such as bladder cancer, head and neck cancer, and GI malignancies. ${ }^{42-45}$

\section{CLINICAL CONSIDERATIONS OF IMMUNE CHECKPOINT BLOCKADE}

Starting with the development of CTLA-4-blocking antibodies, several unique clinical considerations became apparent. The experience with PD-1/PD-L1 checkpoint blockade is less mature, but similar themes continue to emerge.

\section{Patterns of Disease Response: The Immune-Related Response Criteria}

Several patients treated in early studies with CTLA- 4 blockade were noted to have early evidence of disease progression but ultimately achieved disease response. ${ }^{46}$ In a report of some of the first patients treated with CTLA- 4 blockade, the median time it took to achieve response for patients who achieved an ultimate complete response was 30 months. ${ }^{17}$ In one long-term analysis of patients with melanoma who lived at least 4 years after ipilimumab, approximately $25 \%$ had never achieved an outcome better than progressive disease, as traditionally defined by WHO criteria. $^{16}$

In an effort to capture possibly beneficial effects of CTLA-4 therapy beyond those captured by standard radiographic response criteria such as RECIST, investigators proposed alternative response criteria for immunologic checkpoint blockade called the immunerelated response criteria (irRC). ${ }^{47,48}$ In concept, the irRC enable new lesions to be included in the total tumor burden assessment without immediately being considered progressive disease and require confirmation of apparent initial disease progression on a subsequent radiographic assessment (Table 2). In a retrospective study of patients treated with ipilimumab in pooled phase II studies, the irRC were found to correlate with overall survival better than the traditional 


\begin{tabular}{|c|c|c|}
\hline Factor & RECIST & $\operatorname{irRC}$ \\
\hline Measurement of tumor burden & Unidimensional & Bidimensional \\
\hline Complete response & $\begin{array}{l}\text { Disappearance of all target and nontarget lesions; lymph nodes } \\
\text { must regress to }<10 \text {-mm short axis; no new lesions; } \\
\text { requires confirmation }\end{array}$ & Same as for RECIST \\
\hline Partial response & $\begin{array}{l}\geq 30 \% \text { decrease in tumor burden compared with baseline; } \\
\text { requires confirmation }\end{array}$ & $\begin{array}{l}\geq 50 \% \text { decrease in tumor burden compared with baseline; } \\
\text { requires confirmation }\end{array}$ \\
\hline Progressive disease & $\begin{array}{l}\geq 20 \%+5 \text {-mm absolute increase in tumor burden compared } \\
\text { with nadir; progression of nontarget lesions and/or } \\
\text { appearance of new lesions (at any single time point) }\end{array}$ & $\begin{array}{l}\geq 25 \% \text { increase in tumor burden compared with most } \\
\text { recent prior evaluation; new lesions added to tumor } \\
\text { burden; requires confirmation }\end{array}$ \\
\hline Stable disease & $\begin{array}{l}\text { Any response pattern that does not meet criteria for complete } \\
\text { response, partial response, or progressive disease }\end{array}$ & Same as for RECIST \\
\hline
\end{tabular}

WHO response criteria. ${ }^{47}$ The irRC developed from experience with CTLA-4 therapy, but some patients treated with PD-1 agents have similarly shown immune-related patterns of response. ${ }^{32,33}$

Although these atypical late responses can be seen, they are the exception. In one study of 192 patients treated with pembrolizumab, approximately $10 \%$ of patients with progressive disease by irRC at week 12 subsequently achieved some benefit from pembrolizumab therapy as either a response or stable disease. ${ }^{49}$ In a recently presented phase III study of nivolumab versus chemotherapy, of 120 patients who received nivolumab, 10 (8\%) had irRC responses. ${ }^{35}$ This emerging experience with PD-1 atypical responses seems roughly similar to the ipilimumab experience. In pooled phase II data from 123 patients with progression on ipilimumab at the week 12 scan, five patients (4\%) ultimately had an immune-related partial response, and 17 patients (14\%) ultimately achieved stable disease. ${ }^{47}$

The irRC continue to be refined. Because efficacy to PD-1/PD-L1 checkpoint-blocking antibodies is typically made using the unidimensional RECIST criteria, a unidimensional irRC has been proposed. ${ }^{50}$ Ultimately, the role of the irRC as a surrogate end point for efficacy of immune checkpoint blockade will depend on its eventual validation in ongoing randomized controlled prospective trials. If this prospective validation is attained, it will help solidify irRC as a clinical end point acceptable to regulatory authorities.

\section{Immune-Related Adverse Events}

Although immune checkpoint blockade can induce significant antitumor benefits, because checkpoint blockade does not just enhance tumor-specific immune responses, unique adverse effects can occur through nonspecific immunologic activation. Adverse effects from these agents have been termed immune-related adverse events (irAEs) or, occasionally, adverse events of special interest. For clarity and simplicity throughout this review, we denote them all as irAEs. Although irAEs are different in character from adverse events caused by traditional chemotherapy or targeted therapy, the rate of grade 3 or 4 toxicity with immune checkpoint blockade (approximately 10\% to $20 \%$ ) is no greater than that seen with many standard chemotherapy or targeted therapy regimens. ${ }^{51,52}$

IrAEs include dermatologic, GI, hepatic, endocrine, and other less common inflammatory events. Because irAEs likely arise from general immunologic enhancement, temporary immunosuppression with corticosteroids, tumor necrosis factor $\alpha$ antagonists, mycophenolate mofetil, or other agents is often necessary and should follow established algorithms. ${ }^{53}$ Recommendations for treating irAEs come from general clinical consensus because no prospective trials have been conducted to specifically test whether one management strategy is superior. Early recognition and treatment are believed to be important in mitigating severity.

The most common irAE for both CTLA- 4 and PD-1 therapy involves dermatologic toxicity, which is also typically the irAE with the earliest onset, within several weeks (as reviewed for CTLA- 4 by Weber et $\mathrm{al}^{54}$ ). Physical examination findings can consist of a reticular, maculopapular, erythematous rash on the extremities or trunk. ${ }^{55}$ Perhaps more unique to the PD-1 experience, oral mucositis and/or complaints of dry mouth have been reported in a small percentage of patients. ${ }^{34}$ Treatment for most rashes is successful with topical corticosteroids and antipruritics (eg, hydroxyzine, diphenhydramine), and oral corticosteroid rinses and lidocaine have anecdotally been effective in patients with mucositis. More rarely, severe rashes such as StevensJohnson syndrome and toxic epidermal necrolysis have been reported, and if suspected, hospitalization for intravenous corticosteroids, fluid, and electrolyte monitoring is required.

Perhaps the most clinically relevant irAE consists of diarrhea/ colitis, which typically does not present until approximately 6 weeks into treatment after several doses of CTLA- 4 blockade. ${ }^{56}$ The incidence of grade 3 or 4 diarrhea with ipilimumab $3 \mathrm{mg} / \mathrm{kg}$ has been reported to be approximately $10 \%$, but unfortunately, this adverse event has also resulted in treatment-related deaths. ${ }^{14}$ Treatment of moderate symptoms should consist of oral corticosteroids. Hospitalization should be considered for intravenous corticosteroids if symptoms are severe or refractory to oral corticosteroids. Infliximab (5 $\mathrm{mg} / \mathrm{kg}$ once every 2 weeks) can be helpful in corticosteroid-refractory patients. ${ }^{57,58}$ The incidence of grade 3 or 4 diarrhea/colitis with PD-1 agents (approximately $1 \%$ to $2 \%)^{32,33}$ is believed to be much lower than with CTLA-4 agents. Clostridium difficile and other infectious etiologies should be excluded.

Elevations in serum levels of hepatic enzymes (AST and ALT) can be seen with both CTLA-4 and PD-1 blockade, and in almost all cases, these are asymptomatic laboratory abnormalities only. With CTLA-4 blockade, grade 3 or 4 transaminitis is believed to occur less than $10 \%$ of the time, ${ }^{13,14}$ and grade 3 or 4 transaminitis with PD-1 blockade is rare. ${ }^{32,33}$ Patients should be managed with oral corticosteroids or, if refractory, mycophenolate mofetil (500 mg twice daily). Infliximab should not be used because it may contribute to hepatotoxicity. 
Immune checkpoint blockade may also result in endocrinopathies affecting the pituitary, adrenal, and thyroid glands. Clinical symptoms may vary but often involve nonspecific symptoms such as fatigue, headache, and nausea. Diagnosis is usually made by characteristic laboratory findings and/or radiographic changes, such as enlargement of the pituitary gland. ${ }^{59,60}$ Because the pituitary gland also regulates the ovary/testes, pituitary dysfunction may contribute to gonadal dysfunction in women and men of any age. The precise incidence of endocrinopathy is difficult to ascertain because endocrinopathies have been variably monitored and diagnosed in clinical trials. Treatment, as with other irAEs, consists of corticosteroids and, if needed, longer term hormone supplementation such as with levothyroxine or replacement hydrocortisone. Other rare irAEs, such as uveitis, ${ }^{61}$ neurologic adverse effects, ${ }^{62}$ pancreatitis, ${ }^{63}$ hematologic adverse effects, ${ }^{64,65}$ nephritis, ${ }^{66}$ and pneumonitis, ${ }^{32}$ have been described with checkpoint blockade. Pneumonitis is notable because rare treatment-related deaths have been experienced in early clinical studies of PD-1 blockade. ${ }^{32}$

Prolonged immunosuppression, often required to treat irAEs, unfortunately carries the risk of predisposing patients to opportunistic infections. In one case report, a patient treated with ipilimumab who required corticosteroids and infliximab for colitis ultimately developed Aspergillus pneumonia. ${ }^{67}$ Given this risk, in patients receiving prolonged immunosuppression to treat an irAE, such as prednisone $\geq 20 \mathrm{mg}$ per day for at least 4 weeks, we recommend considering prophylaxis against infectious organisms such as Pneumocystis jirovecii following the guidelines established by the National Comprehensive Cancer Network. $^{68}$

Whether irAEs or other exacerbations of autoimmunity occur at a higher rate among patients with underlying autoimmune disorders is unknown because patients with autoimmune disorders were not included in clinical trials of CTLA-4 and PD-1/PD-L1 agents. Nonetheless, several cases have recently been reported of patients with autoimmune disorders successfully being treated with ipilimumab without exacerbation of their underlying autoimmune disorder. ${ }^{69,70}$ Another study, however, indicated that a patient with multiple sclerosis had worsening symptoms after ipilimumab. ${ }^{71}$ Clinicians contemplating this treatment in this patient population should weigh the potential benefits of CTLA- 4 and PD-1/PD-L1 therapy in treating life-threatening malignancy against the theoretical risk of exacerbating an underlying autoimmune disorder.

\section{ONGOING OUESTIONS}

\section{Optimizing Dose and Schedule}

Many questions remain about the optimal dose and schedule of administering immunologic checkpoint blockade. In one phase II dose-finding study of ipilimumab, ipilimumab at $10 \mathrm{mg} / \mathrm{kg}$ compared with $3 \mathrm{mg} / \mathrm{kg}$ was associated with a numerically small but statistically significant higher response rate $(11.1 \% \vee 4.2 \%$, respectively; $P=.0015$ ) but similar rates of disease control. An ongoing randomized study powered for overall survival evaluating ipilimumab $10 \mathrm{mg} / \mathrm{kg}$ versus ipilimumab $3 \mathrm{mg} / \mathrm{kg}$ will more definitively answer this question.

Across all PD-1 agents, multiple studies have shown that doses of PD- 1 agents greater than $1 \mathrm{mg} / \mathrm{kg}$ do not increase efficacy. The largest experience evaluating dosing of a PD-1 antibody was a study of 173 patients with melanoma who were randomly assigned to pembrolizumab $10 \mathrm{mg} / \mathrm{kg}$ or $2 \mathrm{mg} / \mathrm{kg}$ administered every 3 weeks. ${ }^{36}$ Efficacy and safety in both treatment arms were the same. Ongoing phase III studies of pembrolizumab and other PD-1 agents will continue to clarify whether there is a dose-response relationship with PD-1 agents.

The optimal scheduling of CTLA- 4 and PD- 1 antibodies is also not yet known. As per US Food and Drug Administration approval, ipilimumab is delivered every 3 weeks for four total treatments, called induction. In the large registrational ipilimumab study, patients who initially achieved stable disease or a response with acceptable toxicity but who subsequently experienced progression were offered a reinduction course of four additional doses of ipilimumab, with each dose administered 3 weeks apart. ${ }^{14}$ Among the 31 patients who were treated with reinduction ipilimumab in this study, six patients (19\%) achieved a subsequent complete or partial response with no new types of toxicities. ${ }^{72}$ Retreating patients with ipilimumab who experienced some benefit from their initial ipilimumab course with minimal adverse effects is justified.

PD-1/PD-L1 antibodies are administered on a continuous schedule, yet it remains unclear as to whether this continuous dosing is necessary. Many published trials have shown that patients can have persistent responses lasting beyond the cessation of PD-1 therapy. ${ }^{34}$ One patient who had benefit from nivolumab but who later experienced progression reinitiated nivolumab and later regained a response, ${ }^{73}$ similar to the benefits seen in some patients who have received reinduction ipilimumab.

\section{Biomarkers Associated With Disease Outcome}

Treatment with immunologic checkpoint blockade would ideally be matched to patients who are most likely to benefit and least likely to experience adverse effects. The efficacy of CTLA-4 and PD-1 blockade also somewhat varies among different tumor types, and better understanding these differences would enhance the efficacy of this treatment modality. At this time, no single immunologic or tumoral characteristic in a patient has been found to solely determine response to an immunotherapeutic agent.

Both host immunologic and intratumoral factors have been examined with CTLA-4 blockade. In the peripheral blood, CTLA-4 blockade has been shown to result in T-cell activation and proliferation and an increase in antigen-specific immunity. ${ }^{74-76}$ The absolute lymphocyte count has been found to be a specific pharmacodynamic biomarker of ipilimumab, ${ }^{77}$ and the degree of increase in the absolute lymphocyte count has been associated with overall survival in patients with melanoma. ${ }^{77-79}$ The activation marker, inducible costimulator, has also been found to increase during ipilimumab and correlate with benefit. ${ }^{80,81}$ It is possible that these pharmacodynamic biomarkers may ultimately help refine early surrogate assessments of CTLA-4 efficacy and distinguish patient populations for subsequent clinical trials. Intratumorally, a high number of T regulatory cells, the presence of the immunosuppressive enzyme indoleamine 2,3-dioxygenase, and immune-active genetic profile have been associated with benefit from ipilimumab. ${ }^{82,83}$ More recently, whole-exome sequencing of tumors from patients treated with CTLA-4 blockade has revealed mutations that lead to neoantigens, which may be immunologically relevant in responses to immune checkpoint blockade. ${ }^{84,85}$

Although PD-1/PD-L1 plays a role in inhibiting multiple stages of the immune response, $\mathrm{PD}-1 / \mathrm{PD}-\mathrm{L} 1$ blockade is believed to be most relevant in the final stages of the immune response between a $\mathrm{T}$ cell 
and tumor. Thus, most biomarker investigations for PD-1/PD-L1 agents have focused on the tumor microenvironment and, specifically, immunohistochemical expression of one of the ligands for PD-1, PD-L1. Across all studies in multiple tumor types, it seems that patients whose tumors express PD-L1, as detected by immunohistochemical assays, have numerically higher response rates to PD-1/ PD-L1 blockade than patients who do not express PD-L1. ${ }^{32,86,87}$ Nevertheless, patients who do not express PD-L1 can still have impressive responses to PD-1 blockade and should be considered eligible for PD-1-blocking approaches. It remains unclear whether differential expression levels of PD-L1 among various tumor types account for the somewhat different response rates observed. Yet, the explanation for the varying response rates to $\mathrm{PD}-1$ blockade among different tumor types is likely more complex. PD-L1 is inducible and can be upregulated in response to infiltrating immune cells and, possibly, genetic changes within the tumor. ${ }^{88-90}$ Technical considerations of PD-L1 assessments, such as the degree of staining required to be considered positive and whether staining the tumor cells or infiltrating immune cells is relevant, are areas of ongoing research. Until additional knowledge is attained, we do not support using PD-L1 to select patients for PD-1 blockade.

\section{Combination Approaches}

To improve on the number of patients who benefit from immune checkpoint blockade, CTLA-4 and PD-1/PD-L1 antibodies are being combined together and with other anticancer agents such as chemotherapy, targeted therapy, radiotherapy, and other immunotherapy. Many of these combination approaches are built on a robust scientific background, but data from randomized studies are not yet available to suggest that any specific combination approach is more efficacious than single-agent CTLA-4 or PD-1/PD-L1 blockade.

Although traditionally considered immunosuppressive, several preclinical studies indicate that chemotherapy may have immunostimulatory properties, as reviewed by Zitvogel et al. ${ }^{91}$ Some trials in patients with non-small-cell lung cancer and melanoma showed that combinations of chemotherapy with ipilimumab were generally safe, ${ }^{19,92}$ but others have reported an increase in specific adverse effects such as transaminitis. ${ }^{15}$ Dose and schedule may be important in future trials to minimize toxicity and determine whether chemotherapy enhances or inhibits immune checkpoint blockade.

Targeted therapy directed at oncogenic signaling pathways, such as the BRAF mutation in melanoma, is also an attractive partner to combine with immune checkpoint blockade based on preclinical data, as reviewed by Hu-Lieskovan et al. ${ }^{93}$ The combination of ipilimumab and vemurafenib resulted in frequent transaminitis in a phase I study and is not clinically recommended. ${ }^{94}$ The combination of ipilimumab, dabrafenib, and trametinib also was found to be problematic, with frequent colitis, but it is possible that ipilimumab may be able to be combined safely with dabrafenib. ${ }^{95}$ In patients with renal cell carcinoma, the combination of tremelimumab and sunitinib was not found to be tolerable, ${ }^{96}$ and the combination of nivolumab with sunitinib and pazopanib, although generally manageable and associated with significant antitumor activity, was also associated with hepatic and renal toxicity that seemed to be higher than with each single agent alone. ${ }^{97}$

Radiotherapy has been shown to result in a number of potentially beneficial immunologic effects, as reviewed by Formenti and
Demaria. ${ }^{98}$ Preclinical studies also suggest the possibility that radiotherapy can enhance the efficacy of CTLA-4 and PD-1 blockade. ${ }^{99,100}$ Although some clinical cases and retrospective series suggest that radiotherapy may have enhanced the efficacy of immune checkpoint blockade in patients and is generally believed to be safe, ${ }^{101-105}$ prospective trials are under way to test this hypothesis, and no data are yet available.

CTLA- 4 blockade has also been administered together with other immunologic agents, such as the indoleamine 2,3-dioxygenase inhibitor INCB024360, ${ }^{106}$ the oncolytic virus talimogene laherparepvec, ${ }^{107}$ and granulocyte-macrophage colony-stimulating factor, ${ }^{108}$ with encouraging early results. We expect subsequent studies involving engineered T-cell-based therapies and checkpoint blockade.

Other promising data involve CTLA-4 combinations with PD-1 blockade. A phase I study of ipilimumab and nivolumab in patients with melanoma resulted in a high durable response rate and impressive overall survival compared with historical data. ${ }^{109,110}$ Although the most recently reported grade 3 or 4 toxicity rate in patients with melanoma was $64 \%$, which is higher than either ipilimumab or nivolumab individually, ${ }^{111}$ the vast majority of these irAEs were asymptomatic laboratory abnormalities of unclear clinical consequence. For example, elevations in amylase or lipase were reported in $21 \%$ of patients, none of whom met clinical criteria for a diagnosis of pancreatitis. The rate of grade 3 or 4 diarrhea was $7 \%$, which is approximately similar to the rate of grade 3 or 4 diarrhea with ipilimumab monotherapy at $3 \mathrm{mg} / \mathrm{kg}$. Whether ipilimumab and nivolumab improve overall survival compared with either nivolumab or ipilimumab alone remains the subject of an ongoing phase III randomized trial, and investigations of the combination of ipilimumab and nivolumab (and tremelimumab and MEDI4736) are ongoing in many other cancers.

\section{CONCLUSION}

Immunotherapy with checkpoint-blocking antibodies targeting CTLA-4 and PD-1/PD-L1 has improved the outlook for patients with a variety of malignancies. Despite the promise of this approach, many questions remain, such as the optimal management of irAEs and how best to evaluate combination approaches to determine whether they will increase the efficacy of CTLA-4 or PD-1/PD-L1 blockade alone. Themes from the experience with CTLA-4 and PD-1/PD-L1 will likely be relevant for investigations of novel immunologic checkpoints in the future.

\section{AUTHORS' DISCLOSURES OF POTENTIAL CONFLICTS} OF INTEREST

Disclosures provided by the authors are available with this article at www.jco.org.

\section{AUTHOR CONTRIBUTIONS}

Conception and design: All authors

Collection and assembly of data: All authors

Data analysis and interpretation: All authors

Manuscript writing: All authors

Final approval of manuscript: All authors 


\section{REFERENGES}

1. Linsley PS, Brady W, Urnes M, et al: CTLA-4 is a second receptor for the $B$ cell activation antigen B7. J Exp Med 174:561-569, 1991

2. Schneider $H$, Downey J, Smith $A$, et al: Reversal of the TCR stop signal by CTLA-4. Science 313:1972-1975, 2006

3. Riley JL, Mao M, Kobayashi S, et al: Modulation of TCR-induced transcriptional profiles by ligation of CD28, ICOS, and CTLA-4 receptors. Proc Nat Acad Sci USA 99:11790-11795, 2002

4. Qureshi OS, Zheng Y, Nakamura K, et al: Trans-endocytosis of CD80 and CD86: a molecular basis for the cell-extrinsic function of CTLA-4. Science 332:600-603, 2011

5. Krummel MF, Allison JP: CTLA-4 engagement inhibits IL-2 accumulation and cell cycle progression upon activation of resting $T$ cells. J Exp Med 183:2533-2540, 1996

6. Waterhouse $P$, Penninger JM, Timms E, et al: Lymphoproliferative disorders with early lethality in mice deficient in Ctla-4. Science 270:985-988, 1995

7. Tivol EA, Borriello $F$, Schweitzer AN, et al: Loss of CTLA-4 leads to massive lymphoproliferation and fatal multiorgan tissue destruction, revealing a critical negative regulatory role of CTLA-4. Immunity 3:541-547, 1995

8. Leach $D R$, Krummel MF, Allison JP: Enhancement of antitumor immunity by CTLA-4 blockade. Science 271:1734-1736, 1996

9. van Elsas $A$, Hurwitz AA, Allison JP: Combination immunotherapy of B16 melanoma using anti-cytotoxic $T$ lymphocyte-associated antigen 4 (CTLA-4) and granulocyte/macrophage colony-stimulating factor (GM-CSF)producing vaccines induces rejection of subcutaneous and metastatic tumors accompanied by autoimmune depigmentation. J Exp Med 190:355-366, 1999

10. Hodi FS, Butler M, Oble DA, et al: Immunologic and clinical effects of antibody blockade of cytotoxic T lymphocyte-associated antigen 4 in previously vaccinated cancer patients. Proc Natl Acad Sci USA 105:3005-3010, 2008

11. Phan GQ, Yang JC, Sherry RM, et al: Cancer regression and autoimmunity induced by cytotoxic $T$ lymphocyte-associated antigen 4 blockade in patients with metastatic melanoma. Proc Natl Acad Sci USA 100:8372-8377, 2003

12. Ribas $A$, Camacho LH, Lopez-Berestein G, et al: Antitumor activity in melanoma and anti-self responses in a phase I trial with the anti-cytotoxic T lymphocyte-associated antigen 4 monoclonal antibody CP-675,206. J Clin Oncol 23:8968-8977, 2005

13. Ribas $A$, Kefford R, Marshall MA, et al: Phase III randomized clinical trial comparing tremelimumab with standard-of-care chemotherapy in patients with advanced melanoma. J Clin Oncol 31:616-622, 2013

14. Hodi FS, O'Day SJ, McDermott DF, et al: Improved survival with ipilimumab in patients with metastatic melanoma. N Engl J Med 363:711-723, 2010

15. Robert $C$, Thomas $L$, Bondarenko I, et al: Ipilimumab plus dacarbazine for previously untreated metastatic melanoma. N Engl J Med 364: 2517-2526, 2011

16. Wolchok JD, Weber JS, Maio $M$, et al: Four-year survival rates for patients with metastatic melanoma who received ipilimumab in phase II clinical trials. Ann Oncol 24:2174-2180, 2013

17. Prieto PA, Yang JC, Sherry RM, et al: CTLA-4 blockade with ipilimumab: Long-term follow-up of
177 patients with metastatic melanoma. Clin Cancer Res 18:2039-2047, 2012

18. Schadendorf D, Hodi F, Robert C, et al: Pooled analysis of long-term survival data from phase II and phase III trials of ipilimumab in metastatic or locally advanced, unresectable melanoma. European Cancer Congress 2013 (ECCO-ESMOESTRO), September 27-October 1, 2013, Amsterdam, the Netherlands (abstr 24)

19. Lynch TJ, Bondarenko I, Luft A, et al: Ipilimumab in combination with paclitaxel and carboplatin as first-line treatment in stage IIIB/IV non-small-cell lung cancer: Results from a randomized, double-blind, multicenter phase II study. J Clin Oncol 30:2046-2054, 2012

20. Le DT, Lutz E, Uram JN, et al: Evaluation of ipilimumab in combination with allogeneic pancreatic tumor cells transfected with a GM-CSF gene in previously treated pancreatic cancer. J Immunother 36:382-389, 2013

21. Slovin SF, Higano CS, Hamid O, et al: Ipilimumab alone or in combination with radiotherapy in metastatic castration-resistant prostate cancer: Results from an open-label, multicenter phase I/II study. Ann Oncol 24:1813-1821, 2013

22. Kwon ED, Drake CG, Scher HI, et al: Ipilimumab versus placebo after radiotherapy in patients with metastatic castration-resistant prostate cancer that had progressed after docetaxel chemotherapy (CA184-043): A multicentre, randomised, double-blind, phase 3 trial. Lancet Oncol 15:700712, 2014

23. Calabrò L, Morra A, Fonsatti E, et al: Tremelimumab for patients with chemotherapy-resistant advanced malignant mesothelioma: An open-label, single-arm, phase 2 trial. Lancet Oncol 14:11041111,2013

24. Ishida Y, Agata Y, Shibahara K, et al: Induced expression of PD-1, a novel member of the immunoglobulin gene superfamily, upon programmed cell death. EMBO J 11:3887-3895, 1992

25. Freeman GJ, Long AJ, Iwai Y, et al: Engagement of the PD-1 immunoinhibitory receptor by a novel B7 family member leads to negative regulation of lymphocyte activation. J Exp Med 192:10271034,2000

26. Keir ME, Liang SC, Guleria I, et al: Tissue expression of PD-L1 mediates peripheral T cell tolerance. J Exp Med 203:883-895, 2006

27. Nishimura $H$, Nose $M$, Hiai $H$, et al: Development of lupus-like autoimmune diseases by disruption of the PD-1 gene encoding an ITIM motif-carrying immunoreceptor. Immunity 11:141151, 1999

28. Nishimura H, Okazaki T, Tanaka Y, et al: Autoimmune dilated cardiomyopathy in PD-1 receptor-deficient mice. Science 291:319-322, 2001

29. Dong H, Strome SE, Salomao DR, et al: Tumor-associated B7-H1 promotes T-cell apoptosis: A potential mechanism of immune evasion. Nat Med 8:793-800, 2002

30. Fanoni $D$, Tavecchio $S$, Recalcati $S$, et al: New monoclonal antibodies against B-cell antigens: Possible new strategies for diagnosis of primary cutaneous B-cell lymphomas. Immunol Lett 134: 157-160, 2011

31. Infante JR, Powederly JD, Burris, Howard A. et al: Clinical and pharmacodynamic (PD) results of a phase I trial with AMP-224 (B7-DC FC) that binds to the PD-1 receptor. J Clin Oncol 31, 2013 (suppl; abstr 3044)

32. Topalian SL, Hodi FS, Brahmer JR, et al: Safety, activity, and immune correlates of anti-PD-1 antibody in cancer. N Engl J Med 366:2443-2454, 2012
33. Hamid O, Robert C, Daud A, et al: Safety and tumor responses with lambrolizumab (anti-PD-1) in melanoma. N Engl J Med 369:134-144, 2013

34. Topalian SL, Sznol M, McDermott DF, et al: Survival, durable tumor remission, and long-term safety in patients with advanced melanoma receiving nivolumab. J Clin Oncol 32:1020-1030, 2014

35. Weber J, Minor D, D'Angelo, S, et al: A phase 3 randomized, open-label study of nivolumab (anti-PD-1; BMS-936558; ONO-4538) versus investigator's choice chemotherapy (ICC) in patients with advanced melanoma after prior anti-CTLA-4 therapy. 2014 European Society of Medical Oncology Congress, Madrid, Spain, September 26-30, 2014 (abstr 728)

36. Robert C, Ribas A, Wolchok JD, et al: Antiprogrammed-death-receptor-1 treatment with pembrolizumab in ipilimumab-refractory advanced melanoma: A randomised dose-comparison cohort of a phase 1 trial. Lancet 384:1109-1117, 2014

37. Berger R, Rotem-Yehudar R, Slama G, et al: Phase I safety and pharmacokinetic study of CT-011, a humanized antibody interacting with PD-1, in patients with advanced hematologic malignancies. Clin Cancer Res 14:3044-3051, 2008

38. Armand $P$, Nagler $A$, Weller EA, et al: Disabling immune tolerance by programmed death-1 blockade with pidilizumab after autologous hematopoietic stem-cell transplantation for diffuse large B-cell lymphoma: Results of an international phase II trial. J Clin Oncol 31:4199-4206, 2013

39. Westin JR, Chu F, Zhang M, et al: Safety and activity of PD1 blockade by pidilizumab in combination with rituximab in patients with relapsed follicular lymphoma: A single group, open-label, phase 2 trial. Lancet Oncol 15:69-77, 2014

40. Butte MJ, Keir ME, Phamduy TB, et al: Programmed death-1 ligand 1 interacts specifically with the B7-1 costimulatory molecule to inhibit $\mathrm{T}$ cell responses. Immunity 27:111-122, 2007

41. Brahmer JR, Tykodi SS, Chow LQ, et al: Safety and activity of anti-PD-L1 antibody in patients with advanced cancer. N Engl J Med 366:24552465, 2012

42. Powles $T$, Vogelzang $N$, Fine $G D$, et al: Inhibition of PD-L1 by MPDL3280A and clinical activity in pts with metastatic urothelial bladder cancer (UBC). J Clin Oncol 32, 2014 (suppl 5s; abstr 5011)

43. Segal NH, Antonia SJ, Brahmer JR, et al: Preliminary data from a multi-arm expansion study of MEDI4736, an anti-PD-L1 antibody. J Clin Oncol 32, 2014 (suppl 5s; abstr 3002)

44. Herbst RS, Gordon MS, Fine GD, et al: A study of MPDL3280A, an engineered PD-L1 antibody in patients with locally advanced or metastatic tumors. J Clin Oncol 31, 2013 (suppl; abstr 3000)

45. Heery CR, O'Sullivan GH, Madan RA, et al: Phase I open-label, multiple ascending dose trial of MSB0010718C, an anti-PD-L1 monoclonal antibody, in advanced solid malignancies. J Clin Oncol 32, 2014 (suppl 5s; abstr 3064)

46. Saenger YM, Wolchok JD: The heterogeneity of the kinetics of response to ipilimumab in metastatic melanoma: Patient cases. Cancer Immun 8:1, 2008

47. Wolchok JD, Hoos A, O'Day S, et al: Guidelines for the evaluation of immune therapy activity in solid tumors: Immune-related response criteria. Clin Cancer Res 15:7412-7420, 2009

48. Hoos $A$, Eggermont $A M$, Janetzki $S$, et al: Improved endpoints for cancer immunotherapy trials. J Natl Cancer Inst 102:1388-1397, 2010

49. Hodi FS, Ribas A, Daud A, et al: Evaluation of immune-related response criteria (irRC) in patients 
(pts) with advanced melanoma (MEL) treated with the anti-PD-1 monoclonal antibody MK-3475. J Clin Oncol 32, 2014 (suppl 5s; abstr 3006)

50. Nishino M, Giobbie-Hurder A, Gargano M, et al: Developing a common language for tumor response to immunotherapy: Immune-related response criteria using unidimensional measurements. Clin Cancer Res 19: 3936-3943, 2013

51. Kelly K, Crowley J, Bunn PA Jr, et al: Randomized phase III trial of paclitaxel plus carboplatin versus vinorelbine plus cisplatin in the treatment of patients with advanced non-small-cell lung cancer: A Southwest Oncology Group trial. J Clin Oncol 19:3210-3218, 2001

52. Flaherty KT, Infante JR, Daud A, et al: Combined BRAF and MEK inhibition in melanoma with BRAF V600 mutations. N Engl J Med 367:16941703, 2012

53. Bristol-Myers Squibb: Yervoy (ipilimumab): Immune-mediated adverse reaction management guide. https://www.hcp.yervoy.com/pdf/rems-managementguide.pdf

54. Weber JS, Kähler KC, Hauschild A: Management of immune-related adverse events and kinetics of response with ipilimumab. J Clin Oncol 30: 2691-2697, 2012

55. Lacouture ME, Wolchok JD, Yosipovitch G, et al: Ipilimumab in patients with cancer and the management of dermatologic adverse events. J Am Acad Dermatol 71:161-169, 2014

56. Weber JS, Dummer R, de Pril V, et al: Patterns of onset and resolution of immune-related adverse events of special interest with ipilimumab: Detailed safety analysis from a phase 3 trial in patients with advanced melanoma. Cancer 119: 1675-1682, 2013

57. Pagès C, Gornet JM, Monsel G, et al: Ipilimumab-induced acute severe colitis treated by infliximab. Melanoma Res 23:227-230, 2013

58. Minor DR, Chin K, Kashani-Sabet M: Infliximab in the treatment of anti-CTLA4 antibody (ipilimumab) induced immune-related colitis. Cancer Biother Radiopharm 24:321-325, 2009

59. Blansfield JA, Beck KE, Tran K, et al: Cytotoxic T-lymphocyte-associated antigen-4 blockage can induce autoimmune hypophysitis in patients with metastatic melanoma and renal cancer. J Immunother 28:593-598, 2005

60. Dillard T, Yedinak CG, Alumkal J, et al: AntiCTLA-4 antibody therapy associated autoimmune hypophysitis: Serious immune related adverse events across a spectrum of cancer subtypes. Pituitary 13:29-38, 2010

61. Robinson MR, Chan CC, Yang JC, et al: Cytotoxic T lymphocyte-associated antigen 4 blockade in patients with metastatic melanoma: A new cause of uveitis. J Immunother 27:478-479, 2004

62. Wilgenhof $S$, Neyns B: Anti-CTLA-4 antibodyinduced Guillain-Barré syndrome in a melanoma patient. Ann Oncol 22:991-993, 2011

63. Di Giacomo AM, Danielli R, Guidoboni M, et al: Therapeutic efficacy of ipilimumab, an antiCTLA-4 monoclonal antibody, in patients with metastatic melanoma unresponsive to prior systemic treatments: Clinical and immunological evidence from three patient cases. Cancer Immunol Immunother 58:1297-1306, 2009

64. Akhtari M, Waller EK, Jaye DL, et al: Neutropenia in a patient treated with ipilimumab (antiCTLA-4 antibody). J Immunother 32:322-324, 2009

65. Gordon 1O, Wade T, Chin $K$, et al: Immunemediated red cell aplasia after anti-CTLA-4 immunotherapy for metastatic melanoma. Cancer Immunol Immunother 58:1351-1353, 2009
66. Izzedine H, Gueutin V, Gharbi C, et al: Kidney injuries related to ipilimumab. Invest New Drugs 32:769-773, 2014

67. Kyi C, Hellmann MD, Wolchok JD, et al: Opportunistic infections in patients treated with immunotherapy for cancer. J Immunother Cancer 2:19, 2014

68. National Comprehensive Cancet Network: Infections. http://www.nccn.org/professionals/physician_gls/ pdf/infections.pdf

69. Pedersen $M$, Andersen $R$, Nørgaard $P$, et al: Successful treatment with Ipilimumab and Interleukin-2 in two patients with metastatic melanoma and systemic autoimmune disease. Cancer Immunol Immunother 63: 1341-1346, 2014

70. Kyi C, Carvajal RD, Wolchok JD, et al: Ipilimumab in patients with melanoma and autoimmune disease. J Immunother Cancer 2:35, 2014

71. Gettings EJ, Hackett CT, Scott TF: Severe relapse in a multiple sclerosis patient associated with ipilimumab treatment of melanoma. Mult Scler lepub ahead of print on September 25, 2014

72. Robert $C$, Schadendorf $D$, Messina $M$, et al: Efficacy and safety of retreatment with ipilimumab in patients with pretreated advanced melanoma who progressed after initially achieving disease control. Clin Cancer Res 19:2232-2239, 2013

73. Lipson EJ, Sharfman WH, Drake CG, et al: Durable cancer regression off-treatment and effective reinduction therapy with an anti-PD-1 antibody. Clin Cancer Res 19:462-468, 2013

74. Wang W, Yu D, Sarnaik AA, et al: Biomarkers on melanoma patient $T$ cells associated with ipilimumab treatment. J Transl Med 10:146, 2012

75. Ribas A, Benz MR, Allen-Auerbach MS, et al: Imaging of CTLA4 blockade-induced cell replication with (18)F-FLT PET in patients with advanced melanoma treated with tremelimumab. J Nucl Med 51: 340-346, 2010

76. Yuan J, Adamow M, Ginsberg BA, et al: Integrated NY-ESO-1 antibody and CD8+ T-cell responses correlate with clinical benefit in advanced melanoma patients treated with ipilimumab. Proc Natl Acad Sci USA 108:16723-16728, 2011

77. Postow MA, Chasalow SD, Yuan J, et al: Pharmacodynamic effect of ipilimumab on absolute lymphocyte count (ALC) and association with overall survival in patients with advanced melanoma. J Clin Oncol 31, 2013 (suppl; abstr 9052)

78. Delyon J, Mateus C, Lefeuvre D, et al: Experience in daily practice with ipilimumab for the treatment of patients with metastatic melanoma: An early increase in lymphocyte and eosinophil counts is associated with improved survival. Ann Oncol 24:1697-1703, 2013

79. Simeone E, Gentilcore G, Giannarelli D, et al: Immunological and biological changes during ipilimumab treatment and their potential correlation with clinical response and survival in patients with advanced melanoma. Cancer Immunol Immunother 63:675-683, 2014

80. Carthon BC, Wolchok JD, Yuan J, et al: Preoperative CTLA-4 blockade: Tolerability and immune monitoring in the setting of a presurgical clinical trial. Clin Cancer Res 16:2861-2871, 2010

81. Ng Tang D, Shen $Y$, Sun J, et al: Increased frequency of ICOS + CD4 T cells as a pharmacodynamic biomarker for anti-CTLA-4 therapy. Cancer Immunol Res 1:229-234, 2013

82. Ji RR, Chasalow SD, Wang $L$, et al: An immune-active tumor microenvironment favors clinical response to ipilimumab. Cancer Immunol Immunother 61:1019-1031, 2012
83. Hamid $O$, Schmidt $H$, Nissan $A$, et al: $A$ prospective phase II trial exploring the association between tumor microenvironment biomarkers and clinical activity of ipilimumab in advanced melanoma. J Transl Med 9:204, 2011

84. van Rooij N, van Buuren MM, Philips D, et al: Tumor exome analysis reveals neoantigen-specific T-cell reactivity in an ipilimumab-responsive melanoma. J Clin Oncol 31:e439-e442, 2013

85. Snyder A, Makarov V, Merghoub $T$, et al: Exome sequencing reveals a genetic basis underlying clinical response to CTLA-4 blockade. N Engl J Med 371:2189-2199, 2014

86. Weber JS, Kudchadkar RR, Yu B, et al: Safety, efficacy, and biomarkers of nivolumab with vaccine in ipilimumab-refractory or -naive melanoma. J Clin Oncol 31:4311-4318, 2013

87. Grosso J, Horak CE, Inzunza D, et al: Association of tumor PD-L1 expression and immune biomarkers with clinical activity in patients (pts) with advanced solid tumors treated with nivolumab (antiPD-1; BMS-936558; ONO-4538). J Clin Oncol 31, 2013 (suppl; abstr 3016)

88. Taube JM, Klein A, Brahmer JR, et al: Association of PD-1, PD-1 ligands, and other features of the tumor immune microenvironment with response to anti-PD-1 therapy. Clin Cancer Res 20: 5064-5074, 2014

89. Atefi M, Avramis E, Lassen A, et al: Effects of MAPK and PI3K pathways on PD-L1 expression in melanoma. Clin Cancer Res 20:3446-3457, 2014

90. Parsa AT, Waldron JS, Panner A, et al: Loss of tumor suppressor PTEN function increases B7-H1 expression and immunoresistance in glioma. Nat Med 13:84-88, 2007

91. Zitvogel L, Kepp O, Kroemer G: Immune parameters affecting the efficacy of chemotherapeutic regimens. Nat Rev Clin Oncol 8:151-160, 2011

92. Weber J, Hamid O, Amin A, et al: Randomized phase I pharmacokinetic study of ipilimumab with or without one of two different chemotherapy regimens in patients with untreated advanced melanoma. Cancer Immun 13:7, 2013

93. Hu-Lieskovan S, Robert L, Homet Moreno B, et al: Combining targeted therapy with immunotherapy in BRAF-mutant melanoma: Promise and challenges. J Clin Oncol 32:2248-2254, 2014

94. Ribas A, Hodi FS, Callahan M, et al: Hepatotoxicity with combination of vemurafenib and ipilimumab. N Engl J Med 368:1365-1366, 2013

95. Puzanov I, Callahan MK, Linette GP, et al: Phase 1 study of the BRAF inhibitor dabrafenib (D) with or without the MEK inhibitor trametinib (T) in combination with ipilimumab (Ipi) for V600E/K mutation-positive unresectable or metastatic melanoma (MM). J Clin Oncol 32, 2014 (suppl 5s; abstr 2511)

96. Rini BI, Stein M, Shannon $P$, et al: Phase 1 dose-escalation trial of tremelimumab plus sunitinib in patients with metastatic renal cell carcinoma. Cancer 117:758-767, 2011

97. Amin A, Plimack E, Infante JR, et al: Nivolumab (anti-PD-1; BMS-936558, ONO-4538) in combination with sunitinib or pazopanib in patients (pts) with metastatic renal cell carcinoma (mRCC). J Clin Oncol 32, 2014 (suppl 5s; abstr 5010)

98. Formenti SC, Demaria S: Combining radiotherapy and cancer immunotherapy: A paradigm shift. J Natl Cancer Inst 105:256-265, 2013

99. Demaria S, Kawashima N, Yang AM, et al: Immune-mediated inhibition of metastases after treatment with local radiation and CTLA-4 blockade in a mouse model of breast cancer. Clin Cancer Res 11:728-734, 2005 
100. Deng $L$, Liang $H$, Burnette $B$, et al: Irradiation and anti-PD-L1 treatment synergistically promote antitumor immunity in mice. J Clin Invest 124:687695, 2014

101. Postow MA, Callahan MK, Barker CA et al: Immunologic correlates of the abscopal effect in a patient with melanoma. N Engl J Med 366:925-931, 2012

102. Sullivan RJ, Lawrence DP, Wargo JA, et al: Case records of the Massachusetts General Hos pital: Case 21-2013-A 68-year-old man with metastatic melanoma. N Engl J Med 369:173-183, 2013

103. Golden EB, Demaria $S$, Schiff $P B$, et al: An abscopal response to radiation and ipilimumab in a patient with metastatic non-small cell lung cancer. Cancer Immunol Res 1:365-372, 2013

104. Grimaldi AM, Simeone E, Giannarelli D, et al: Abscopal effects of radiotherapy on advanced mel- anoma patients who progressed after ipilimumab immunotherapy. Oncoimmunology 3:e28780, 2014

105. Barker CA, Postow MA, Khan SA, et al: Concurrent radiotherapy and ipilimumab immunotherapy for patients with melanoma. Cancer Immunol Res 1:92-98, 2013

106. Gibney G, Hamid O, Gangadhar $T$, et al: Preliminary results from a phase $1 / 2$ study of INCB024360 combined with ipilimumab (ipi) in patients (pts) with melanoma. J Clin Oncol 32, 2014 (suppl 5s; abstr 3010)

107. Puzanov I, Milhem M, Andtbacka $R$, et al: Primary analysis of a phase $1 \mathrm{~b}$ multicenter trial to evaluate safety and efficacy of talimogene laherparepvec (T-VEC) and ipilimumab (ipi) in previously untreated, unresected stage IIIB-IV melanoma. J Clin Oncol 32, 2014 (suppl 5s; abstr 9029)

108. Hodi FS, Lee S, McDermott D, et al: Multicenter, randomized phase II trial of GM-CSF (GM) plus ipilimumab (Ipi) versus Ipi alone in metastatic melanoma: E1608. J Clin Oncol 31, 2013 (suppl; abstr CRA9007)

109. Wolchok JD, Kluger $H$, Callahan MK, et al: Nivolumab plus ipilimumab in advanced melanoma. N Engl J Med 369:122-133, 2013

110. Sznol M, Kluger $H$, Callahan $M K$, et al: Survival, response duration, and activity by BRAF mutation (MT) status of nivolumab (NIVO, antiPD-1, BMS-936558, ONO-4538) and ipilimumab (IPI) concurrent therapy in advanced melanoma (MEL). J Clin Oncol 32, 2014 (suppl 5s; abstr LBA9003)

111. Kluger H, Sznol M, Callahan M, et al: Survival, response duration, and activity by BRAF mutation (MT) status in a phase 1 trial of nivolumab (anti-PD-1, BMS-936558, ONO-4538) and ipilimumab (IPI) concurrent therapy in advanced melanoma (MEL). Ann Oncol 25:iv374-iv393, 2014 (suppl 4)

\section{Best of ASCO Meetings}

Mark your calendar for the 2015 Best of ASCO Meetings, scheduled for July 31 and August 1, 2015, in Boston, MA; August 7 and 8, 2015, in San Francisco, CA; and August 28 and 29, 2015, in Chicago, IL. Featuring practice-changing science and educational highlights from the ASCO Annual Meeting, the Best of ASCO Meetings offer in-depth analysis and discussion of the top scientific abstracts that will directly affect patient care.

For additional details and to register for the meeting, visit boa.asco.org. 


\section{AUTHORS' DISCLOSURES OF POTENTIAL CONFLICTS OF INTEREST}

Immune Checkpoint Blockade in Cancer Therapy

The following represents disclosure information provided by authors of this manuscript. All relationships are considered compensated. Relationships are self-held unless noted. I = Immediate Family Member, Inst = My Institution. Relationships may not relate to the subject matter of this manuscript. For more information about ASCO's conflict of interest policy, please refer to www.asco.org/rwc or jco.ascopubs.org/site/ifc.

\section{Michael A. Postow}

Consulting or Advisory Role: Bioconnections LLC, FirstWord, Millennium Takeda, Amgen

Research Funding: Bristol-Myers Squibb (Inst)

Travel, Accommodations, Expenses: Bristol-Myers Squibb

Margaret K. Callahan

Employment: Bristol-Myers Squibb (I)

Consulting or Advisory Role: Kiowa Hakko Kiran, GlaxoSmithKline

Research Funding: Bristol-Myers Squibb

\section{Jedd D. Wolchok}

Honoraria: EMD Serono, Janssen Oncology

Consulting or Advisory Role: Bristol-Myers Squibb, Merck,

MedImmune, Ziopharm, Polynoma, Polaris, Jounce, GlaxoSmithKline Research Funding: Bristol-Myers Squibb (Inst), MedImmune (Inst), GlaxoSmithKline (Inst), Merck (Inst)

Patents, Royalties, Other Intellectual Property: I am a co-inventor on an issued patent for DNA vaccines for treatment of cancer in companion animals

Travel, Accommodations, Expenses: Bristol-Myers Squibb 